\title{
Coherent $e^{+} e^{-}$production at very low transverse momentum at STAR
}

\author{
Chi Yang ${ }^{1, \star}$ For the STAR Collaboration \\ ${ }^{1}$ Shanda Nanlu 27\#, Shandong University, Jinan, Shandong Province, China 250100
}

\begin{abstract}
We report the measurements of $e^{+} e^{-}$pair production at very low $e^{+} e^{-}$pair transverse momentum $\left(p_{T}<0.15 \mathrm{GeV} / c\right)$ in $\mathrm{Au}+\mathrm{Au}$ collisions at $\sqrt{s_{N N}}=200 \mathrm{GeV}$ and $\mathrm{U}+\mathrm{U}$ collisions at $\sqrt{s_{N N}}=193 \mathrm{GeV}$ using the STAR detector at the Relativistic Heavy Ion Collider. In 60-80\% centrality, significant excesses are observed with respect to hadronic cocktails in both $\mathrm{Au}+\mathrm{Au}$ and $\mathrm{U}+\mathrm{U}$ collisions. These excess yields can not be explained by a theoretical model calculation incorporating in-medium broadened $\rho$ spectral function. Additionally, the $p_{T}^{2}$ distribution for excess yield is shown and found to be exponential at very low $p_{T}$.
\end{abstract}

\section{Introduction}

The $e^{+} e^{-}$pair production provides a unique observable to study the fundamental properties of the hot and dense medium created in ultra-relativistic heavy-ion collisions. Those pairs are produced during all stages of the collisions and from all forms of the created matter. Due to only electromagnetic interactions with this matter, they can convey information about the dynamics of the entire time evolution of the medium [1]. In the $e^{+} e^{-}$invariant mass region $M_{l l}<M_{\phi}$ (Low Mass Region, LMR) the production is dominated by in-medium decay of vector mesons $(\rho, \omega$, and $\phi)$. The broadened $\rho$ model calculation which is linked to chiral symmetry restoration can describe the modified $\rho$ spectra in this region. In intermediate mass region (IMR, $M_{\phi}<M_{l l}<M_{J / \psi}$ ) the $e^{+} e^{-}$pair production consists of two parts: thermal radiation of the medium and semi-leptonic decay.

Recently, the ALICE collaboration reported a significant excess at very low $p_{T}$ in its study of $\mathrm{J} / \psi$ yield in peripheral $\mathrm{Pb}+\mathrm{Pb}$ collisions at $\sqrt{s_{N N}}=2.76 \mathrm{TeV}$ via $\mu^{+} \mu^{-}$channel [2]. The STAR collaboration also observed similar behavior for $\mathrm{J} / \psi$ in peripheral $\mathrm{Au}+\mathrm{Au}$ collisions at $\sqrt{s_{N N}}=200 \mathrm{GeV}$ and $\mathrm{U}+\mathrm{U}$ collisions at $\sqrt{s_{N N}}=193 \mathrm{GeV}$ at $p_{T}<0.15 \mathrm{GeV} / c$ via $e^{+} e^{-}$channel [3]. These results via dilepton channels can not be explained by hadronic production mechanisms while coherent photoproduction and photon-photon interaction mechanism in UPC (ultra-peripheral collisions) can describe some observables. In this case, with impact parameter larger than twice of the nucleus radius, virtual photons emitted by one nucleus can fluctuate into quark-antiquark pairs, and then coherently interact with the other nucleus to produce vector mesons. The other process, photon-photon interaction, will also produce dilepton pairs like $e^{+} e^{-}$pairs. It is necessary to measure the $e^{+} e^{-}$production in a wide mass range including vector meson dominant region and beyond.

\footnotetext{
^e-mail: chiyang@sdu.edu.cn
} 


\section{Low $p_{T} e^{+} e^{-}$production}

\subsection{Analysis}

The data used in this analysis are from $\mathrm{Au}+\mathrm{Au}$ collisions at $\sqrt{s_{N N}}=200 \mathrm{GeV}$ and $\mathrm{U}+\mathrm{U}$ collisions at $\sqrt{s_{N N}}=193 \mathrm{GeV}$. About 720 million $\mathrm{Au}+\mathrm{Au}$ and 270 million $\mathrm{U}+\mathrm{U}$ minimum bias ( $0-80 \%$ centrality) events passed data quality assurances and vertex selections in total. The collision vertex was required to be within $30 \mathrm{~cm}$ of the mean of the vertex distribution along the beam line, nominally at the center of the Time Projection Chamber (TPC). In the plane perpendicular to the beam line, the collision vertex was selected within $2 \mathrm{~cm}$ of the beam line.

The main subsystems used for electron identification were the TPC and the Time-of-Flight (TOF). With the selection requirements on the particle energy loss, $\mathrm{dE} / \mathrm{dx}$, measured by the TPC and particle velocity, $\beta$, measured by the TOF, high purity electron samples were obtained [4]. The electron purity (fraction of true electrons in the identified electron sample) was about $95 \%$ in $\mathrm{Au}+\mathrm{Au}$ minimum bias collisions on average and was $p_{T}$ dependent from 0.2 to $2.0 \mathrm{GeV} / \mathrm{c}$ [5]. The details for $e^{+} e^{-}$ production can be found in Ref. [5].

\subsection{Results}

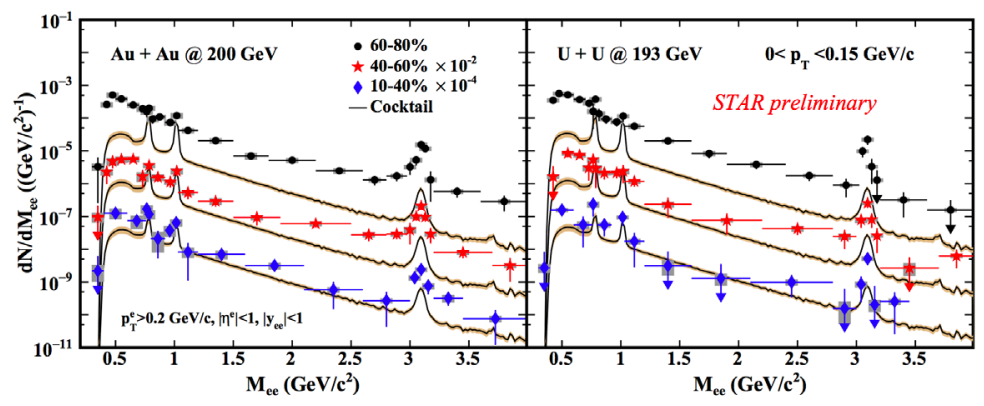

Figure 1. (Color online) The efficiency corrected low $p_{T}(<0.15 \mathrm{GeV} / c) e^{+} e^{-}$pair invariant mass distributions from $\mathrm{Au}+\mathrm{Au}$ collisions at $\sqrt{s_{N N}}=200 \mathrm{GeV}$ (left) and $\mathrm{U}+\mathrm{U}$ collisions at $\sqrt{s_{N N}}=193 \mathrm{GeV}$ (right). The distributions for three different centralities $60-80 \%, 40-60 \%$, and 10-40\% are shown. For each centrality, the corresponding hadronic cocktail contribution is shown as a solid black line with the uncertainty as a shaded band. Statistical and systematic uncertainties on the data points are shown as bars and shaded boxes, respectively.

Figure 1 shows the $e^{+} e^{-}$invariant mass, $M_{e e}$, distribution at $p_{T}<0.15 \mathrm{GeV} / c$ from $\mathrm{Au}+\mathrm{Au}$ collisions at $\sqrt{s_{N N}}=200 \mathrm{GeV}$ and $\mathrm{U}+\mathrm{U}$ collisions at $\sqrt{s_{N N}}=193 \mathrm{GeV}$. The contributions from $\rho$ meson are excluded from the hadronic cocktail simulations for comparison to model calculations. In 60$80 \%$ centrality $\mathrm{Au}+\mathrm{Au}$ and $\mathrm{U}+\mathrm{U}$ collisions, there are significant excesses observed with respect to the corresponding hadronic cocktails [5]. Compared to those in 60-80\% centrality, the enhancement factors in $40-60 \%$ are less significant while in $10-40 \%$ the data are consistent with the hadronic cocktail within uncertainties for $M_{e e}>0.76 \mathrm{GeV} / c^{2}$. The low $p_{T}(<0.15 \mathrm{GeV} / c) e^{+} e^{-}$excess yields with respect to the hadronic cocktail are shown in Fig. 2 from $\mathrm{Au}+\mathrm{Au}$ and $\mathrm{U}+\mathrm{U}$ collisions in $60-80 \%$ centrality. Theoretical calculations [6] expected from in-medium modified $\rho$ production, QGP thermal radiation, and the sum of the two are shown for comparison. These calculations fail to explain the excess. Additional production mechanisms should be involved in this distribution. 


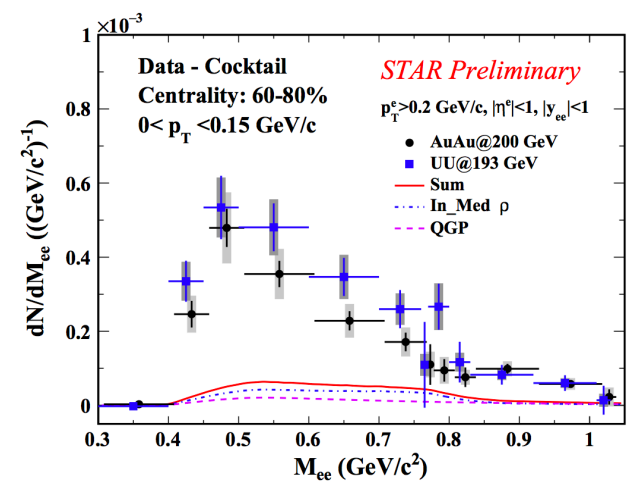

Figure 2. (Color online) The low $p_{T}(<0.15 \mathrm{GeV} / c) e^{+} e^{-}$excess yield with respect to the hadronic cocktail for $\mathrm{Au}+\mathrm{Au}$ and $\mathrm{U}+\mathrm{U}$ collisions in $60-80 \%$ centrality. Statistical and systematic uncertainties on the data points are shown as bars and shaded boxes, respectively. Theoretical calculations expected from in medium modified $\rho$ production, QGP thermal radiation, and the sum of the two are shown for comparison.

Figure 3 shows the $d N / d p_{T}$ distribution as a function of $p_{T}$ for $e^{+} e^{-}$pairs in different mass regions from $\mathrm{Au}+\mathrm{Au}$ and $\mathrm{U}+\mathrm{U}$ collisions. Compared to distributions from hadronic cocktail, clear excesses are observed in $p_{T}<0.15 \mathrm{GeV} / c$. For the $p_{T}$ range above $0.15 \mathrm{GeV} / c$, the hadronic cocktail distributions are consistent with the data within uncertainties. This distribution is similar to that in ultra-peripheral collisions [7].

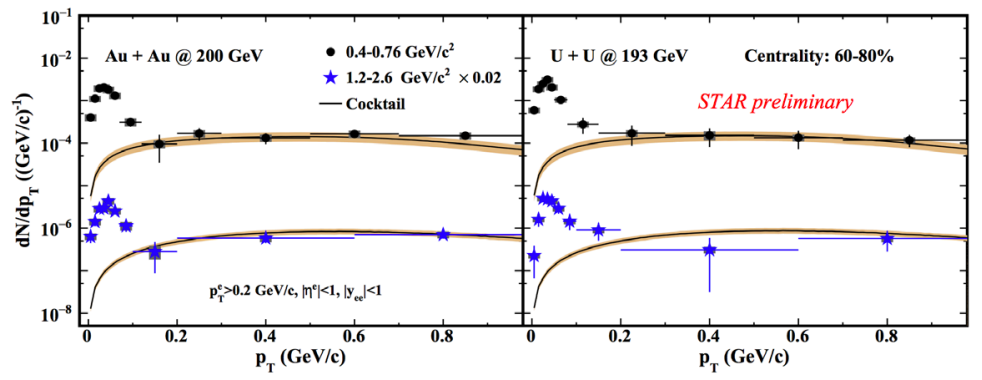

Figure 3. (Color online) The $d N / d p_{T}$ distribution as a function of $p_{T}$ for $e^{+} e^{-}$pairs in different mass region for $\mathrm{Au}+\mathrm{Au}$ collisions at $\sqrt{s_{N N}}=200 \mathrm{GeV}$ (left) and $\mathrm{U}+\mathrm{U}$ collisions at $\sqrt{s_{N N}}=193 \mathrm{GeV}$ (right). For each mass region, the corresponding hadronic cocktail distribution is shown in solid black line with the uncertainty shown as shaded band. Statistical and systematic uncertainties on the data points are shown as bars and shaded boxes, respectively.

An exponential function $\left(A e^{-B p_{T}^{2}}\right)$ is used to fit each $p_{T}^{2}$ distribution in Figure 4. The first data point is excluded from the fit to avoid the shift of the slope because of a predicted dropoff based on quantum electrodynamics calculation [8] (photon-photon process dominant) or a possible destructive interference effect (photon-nucleus process dominant) at very small $p_{T}^{2}$ [9]. The slope parameters from $\mathrm{U}+\mathrm{U}$ collisions are systematically larger than those from $\mathrm{Au}+\mathrm{Au}$ collisions in the same mass region. In the $\rho$-like region, the slope parameter in $\mathrm{Au}+\mathrm{Au}$ peripheral collisions is consistent with 
the coherent photoproduction of $\rho^{0}$ in ultra-peripheral $\mathrm{Au}+\mathrm{Au}$ collisions [9]. Since the excess in the $\rho$-like region is mainly from photon-photon interaction while UPC $\rho^{0}$ comes from photon-nucleus process, this consistency might be accidental.

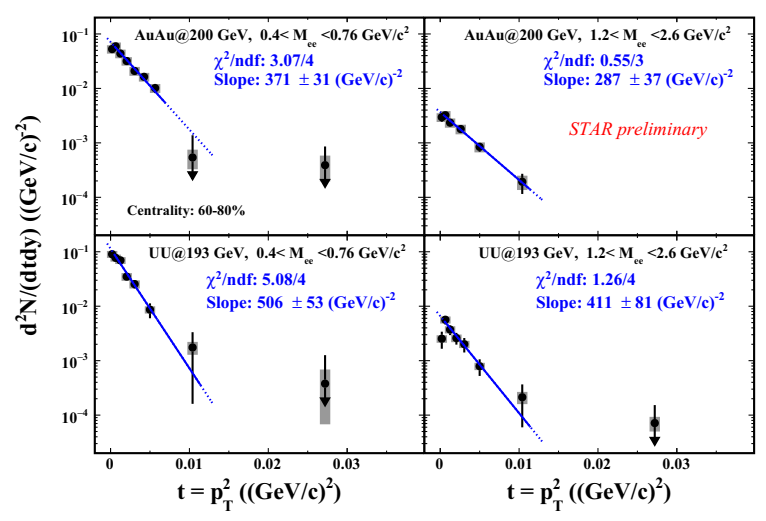

Figure 4. (Color online) The $p_{T}^{2}$ distributions of excess yields in different mass regions for 60-80\% centrality $\mathrm{Au}+\mathrm{Au}$ (upper plots) and $\mathrm{U}+\mathrm{U}$ (lower plots) collisions. The systematic uncertainties of the data are shown as gray boxes.

\section{Summary}

We report the measurements of $e^{+} e^{-}$pair production at very low transverse momentum $\left(p_{T}<0.15\right.$ $\mathrm{GeV} / c)$ in $\mathrm{Au}+\mathrm{Au}$ collisions at $\sqrt{s_{N N}}=200 \mathrm{GeV}$ and $\mathrm{U}+\mathrm{U}$ collisions at $\sqrt{s_{N N}}=193 \mathrm{GeV}$ at STAR. The $e^{+} e^{-}$pair yields in a wider mass region are significantly enhanced with respect to the hadronic cocktail in $60-80 \%$ centrality in both $\mathrm{Au}+\mathrm{Au}$ and $\mathrm{U}+\mathrm{U}$ collisions. These excesses are only observed in the very low $p_{T}$ region $(<0.15 \mathrm{GeV} / c)$.

\section{Acknowledgement}

The author is supported partially by the NNSFC, China under grant number 11505181 .

\section{References}

[1] G. David, R. Rapp, Z. Xu, Phys. Rep. 462, 176 (2008)

[2] J.Adam, et al.,(ALICE Collaboration), Phys. Rev. Lett. 116, 222301 (2016)

[3] W.Zha, J. Phys. Conf. Ser. 779, 12039 (2017)

[4] M. Shao, et al., Nucl. Instrum. Methods A 558, 419 (2006)

[5] L. Adamczyk, et al., (STAR Collaboration), Phys. Rev. C 92, 024912 (2015); Phys. Rev. C (90, 064904 (2014)

[6] R.Rapp, private communication

[7] L. Adamczyk, et al., (STAR Collaboration), arXiv:1702.07705

[8] J. Adams, et al., (STAR Collaboration), Phys. Rev. C 70, 031902 (2004)

[9] B. I. Abelev, et al., (STAR Collaboration), Phys. Rev. C 77, 034910 (2008); Phys. Rev. Lett. 102, 112301 (2009) 